\title{
Análisis de las variables críticas de un sistema productivo a través de la Dinámica de Sistemas
}

\author{
The Critical Variables Analysis of Production System Through System \\ Dynamic \\ Laura Angélica Mejía Ospina, Oscar Arroyave Contreras, Rodrigo Andrés González Bernal \\ Ingeniería Industrial, Unidad Central del Valle del Cauca, Tuluá, Colombia \\ Correo-e: lamejia@uceva.edu.co
}

\begin{abstract}
Resumen - Un sistema productivo que presente falencias en la administración de sus procesos de almacenamiento de inventario y materiales puede ser representado con la metodología de Dinámica de Sistemas, involucrando las variables críticas del mismo y generando un análisis de sensibilidad de acuerdo con diferentes alternativas de decisión, que busquen mejorar sus condiciones de productividad actuales. El artículo expone el resultado de un trabajo de investigación propuesto para una microempresa que presenta bajos índices de productividad total, y, a través de un modelo de simulación se pueden analizar diferentes propuestas de decisión para su mejoramiento.
\end{abstract}

Palabras clave-, Dinámica de Sistemas, Proceso Productivo, Simulación, Variable Crítica.

Abstract- A productive system that shows shortcomings in its
management of storage and material process can be represented
with System Dynamics methodology, involving critical variables
and generating sensitivity analysis according to different decision
alternatives, which seek to improve their current productivity.
This paper presents the results of researching work proposed for
a small business which exposes lower total productivity levels
and, through a simulation model can analyze different proposed
decisions for improvement.

Key Word - System Dynamic, Productive Process, Simulation, Critical Variable.

\section{INTRODUCCIÓN}

La administración de los sistemas productivos en empresas de producción de bienes trae diversas complejidades. Este artículo presenta un estudio realizado en una microempresa del sector producción de calzado deportivo de la ciudad de Tuluá, en donde se expone una serie de problemáticas en la administración de operaciones, específicamente en la gestión y control del almacenamiento de materias primas, inventario de producto final, precio de venta, entre otras variables directamente relacionadas con los costos de producción.

El escenario de estudio del presente artículo se expone en una línea de producción de una microempresa de calzado deportivo en la ciudad de Tuluá. Este proceso productivo viene presentando fallas, relacionadas con planeación de la producción, gestión de inventarios y tiempo de producción; lo que se refleja en el indicador de productividad en costos, en donde, para los últimos 5 meses se ha presentado amplia variabilidad en este indicador, mostrando valores negativos en su medición de productividad total (ver gráfico 1).

De acuerdo con lo anterior surge la necesidad de explorar diferentes alternativas de decisión, que involucre el análisis de las variables críticas del proceso productivo. Para ello se hizo uso de la Dinámica de Sistemas, la cual se expone como una metodología que permite recrear el comportamiento de sistemas, con variables de interés que interactúan entre sí, para analizar su estructura. Esta metodología se utiliza como método para entender el comportamiento no lineal de sistemas complejos [1]. Ofrece también una serie de pasos para elaborar modelos de simulación, validarlos y estudiar el impacto de una variable de interés en relación con un cambio determinado por otra.

En el presente artículo se pretende dar evidencia del planteamiento de un modelo de simulación, aplicando la metodología de Dinámica de Sistemas, para representar un sistema real de producción, y acorde a esto plantear diferentes escenarios de mejora, que permitan evaluar alternativas diferentes y su impacto sus variables críticas de proceso. 


\section{CONTENIDO}

A. El sistema de estudio

El sistema a estudiar corresponde a la incorporación de variables de producción relacionadas con la problemática que presenta la microempresa en estudio. El gráfico 1 ilustra el comportamiento del indicador de productividad total para los últimos 5 meses:

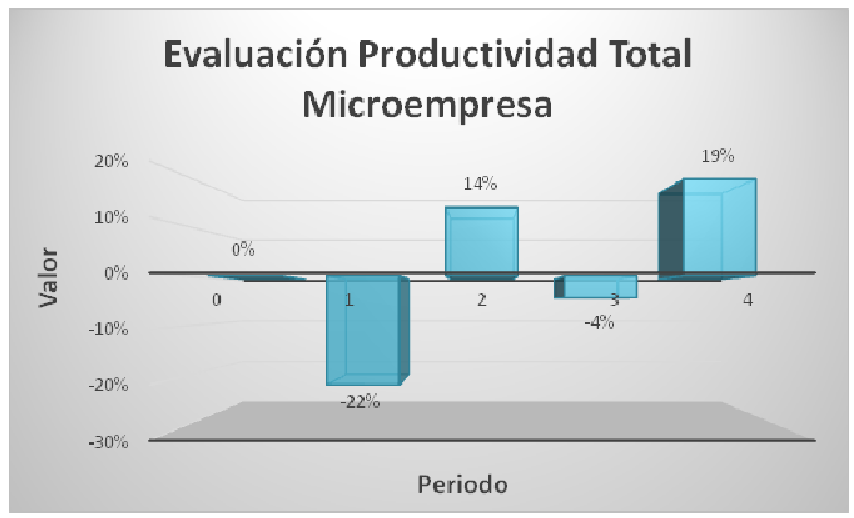

Gráfico 1. Evaluación productividad total de la microempresa de estudio.

El análisis de productividad total se resume en el planteamiento de la razón de los recursos consumidos, frente a los recursos invertidos (salidas/entradas), en donde se plantea una serie de variables de estudio para contemplar la suma de su comportamiento [10]. Para la microempresa se evidencia una disminución en su productividad total, (donde según Sumanth [10], es una medida holística que considera el impacto asociado y simultáneo de todos los recursos en la producción como: la fuerza de trabajo, los materiales, las máquinas, el capital, la energía), para el segundo y cuarto periodo, en cantidades variables. Lo anterior indica una alta variabilidad en el desempeño de la línea de producción, en donde es necesario entrar a evaluar sus variables críticas.

El sistema en estudio pertenece a un sistema de actividad económica relacionada con la fabricación y comercialización de zapatos, en donde se diseña un modelo de simulación para medir su capacidad productiva, teniendo un alcance desde que se tiene la demanda, su proceso productivo, hasta que se contabilizan los ingresos y los egresos, y se determina la utilidad del periodo.

En el proceso del seguimiento hecho al sistema productivo se identifica el cursograma sinóptico (ver figura 1), el cual es un diagrama que evidencia las actividades cronológicas que se realizan para la elaboración del calzado deportivo.

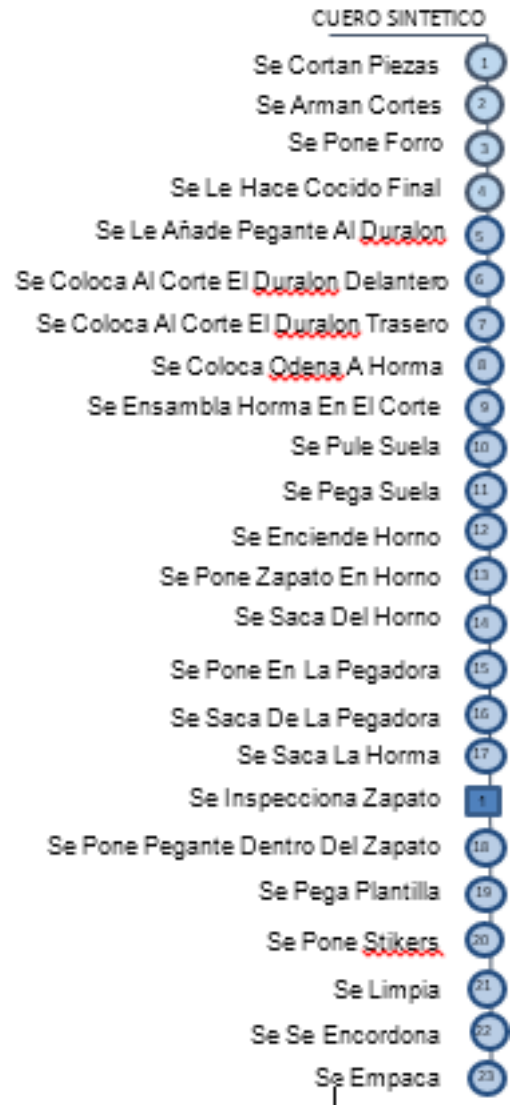

Figura 1. Diagrama Sinóptico Microempresa Calzado Wacky.

En este proceso de obtención de datos, se tuvo registro de 5 meses sobre los costos, gastos e ingresos de la microempresa en estos periodos, datos necesarios para realizar la simulación y que se asemeje al comportamiento real del sistema.

Una de las partes del modelo, se ubica en la compra de materia prima, puesto que lo necesario para la producción en materia prima, no es lo mismo que se pide, ya que cada materia prima cuenta con unidad de venta diferente, es decir, si para la producción de 30 pares de zapatos se necesitan 720 ojales, se hace necesario comprar 1000 que es su unidad de venta.

Por lo tanto el alcance de este modelo, es para las unidades que se tienen en la demanda histórica y el tipo de producto que se consideró el más representativo para la microempresa, es decir, el zapato deportivo con cordones.

\section{B. Metodología}

La metodología propuesta para llevar a cabo la construcción y posterior corrida de la simulación, es el uso de la Dinámica de Sistemas mediante sus herramientas principales como lo son el Diagrama Causal y Forrester, este último se elaborará en el software Stella ${ }^{\circledR}$, en el cual se pudo elaborar el modelo y analizar su comportamiento de acuerdo a la realidad que vive la empresa en la actualidad. 
El pensamiento Sistémico abarca una amplia y heterogénea variedad de métodos, herramientas y principios todos orientados a examinar la interrelación de fuerzas, que forman parte de un proceso común, analizar un contexto como un sistema único. Estos diversos enfoques comparten una idea rectora: la conducta de todos los sistemas sigue ciertos principios comunes, cuya naturaleza apenas se está analizando [2]. Como metodología de análisis cuantitativo de los sistemas, la Dinámica de Sistemas permite tener una perspectiva holística de los elementos que componen un sistema y poder evaluar diferentes alternativas de decisión, teniendo como base el Pensamiento Sistémico. Mediante la recreación de escenarios a pequeña escala, los micromundos, como lo menciona Peter Senge [9], se pueden plantear diferentes variables que demuestran comportamientos de interés para un sistema, se pueden medir, probar y cambiar por medio de modelos de simulación.

El presente artículo ilustra el diseño de un modelo de simulación del sistema productivo en estudio, para un periodo de estudio de 5 meses, con el fin de poder analizar si este se comporta igual a la realidad, y poder así realizar la validación del modelo, de tal forma que sea creíble y confiable.

El estudio incluyó el análisis de variables como: tiempos de producción, las materias primas necesarias, la demanda, el consumo de energía, la política de inventario, costos de producción, tiempo total de producción, entre otros; con los cuales se pudo establecer una relación directa en el estudio de su comportamiento, para la realización del diagrama causa.

Para ello se delimitó concretamente cada parte que interviene en el sistema, separándolo por parte para su mejor entendimiento. El objetivo de este planteamiento, como primer paso de la metodología de análisis desde la Dinámica de Sistemas, corresponde a incorporar un análisis causa-efecto inicial, vinculando todas las variables de interés en el estudio, para interpretar adecuadamente su estructura y su comportamiento.

\section{a. Diagramas Causales}

Para dar origen al modelo, como primera medida se analizó completamente el sistema para luego construir el diagrama causal (figura 2,3,4,5), identificando variables y sus respectivas relaciones, igualmente se asignaron los diferentes tipos de ciclos y los signos que representan la variación ya sea negativa o positiva de una variable con respecto a otra.

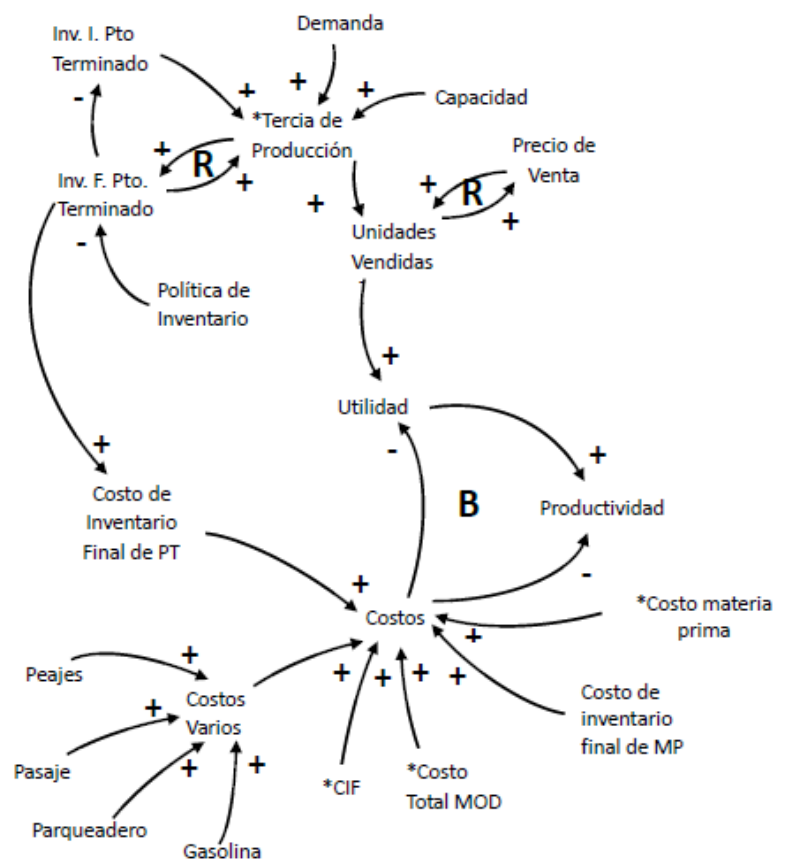

Figura 2. Diagrama Causal Microempresa, relación costos-tercia de producción

La figura 2 ilustra el análisis causa-efecto que presenta la interacción de las variables relacionadas con los costos (directos e indirectos), en el proceso productivo (tercia de producción $)^{1}$. Por ejemplo, entre mayor capacidad se tenga en el sistema productivo, mayor será la tercia de producción (unidades netas de producción por periodo), lo que a su vez resultará en mayor número de unidades vendidas, y redundará en mayor nivel de utilidad, en términos de relación directa. Si aumentan los costos en términos generales, la utilidad disminuirá. Esta última variable es aquella importante de tener en cuenta para el estudio del comportamiento del sistema costos-tercia de producción.

\footnotetext{
${ }^{1}$ Este diagrama es unido por medio de variables que se consideran fantasma, las cuales se pueden identificar con aquellas que tienen un asterisco $\left(^{*}\right)$, éstas son utilizadas con el fin de no saturar el diagrama con líneas, se comportan como una copia de la variable real, (variables fantasma).
} 

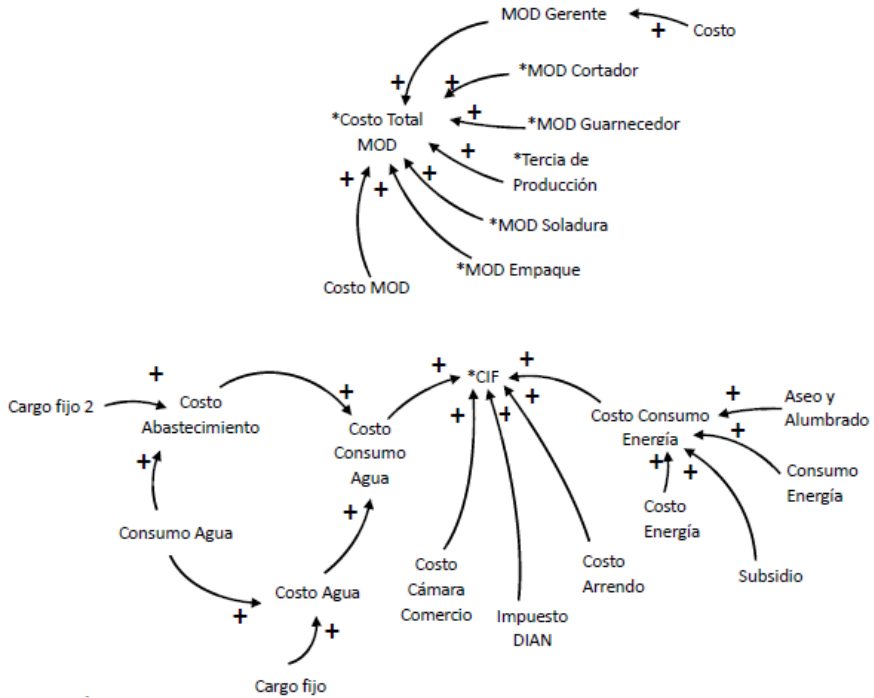

Figura 3. Diagrama Causal Microempresa, relación costos directos costos indirectos.

La figura 3 plantea el análisis del comportamiento de los costos tanto directos como indirectos que parten del proceso de producción. Estos dos diagramas pretenden mostrar la inclusión de la mayoría de variables que intervienen en el cálculo de los costos, y que directamente afectan el estado de resultados por periodo en la microempresa.

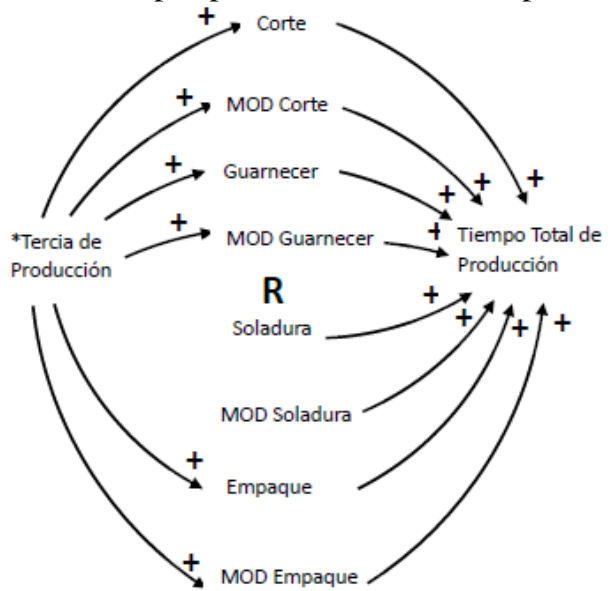

Figura 4. Diagrama Causal Microempresa, relación tiempo de producción - tercia de producción

La cantidad de producción que se establece luego del análisis con los inventarios de producto terminado disponibles para cada periodo, se establece en la figura 4 , en donde se relaciona la tercia de producción, con el tiempo total de producción, de acuerdo al proceso productivo.

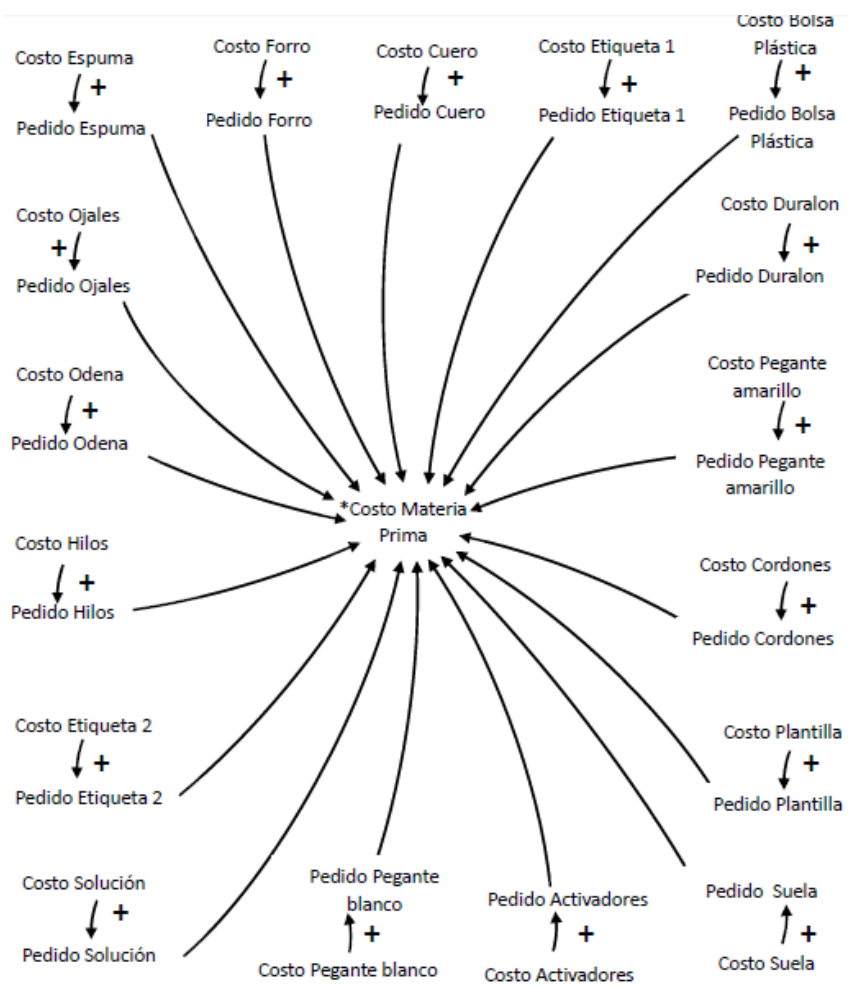

Figura 5. Diagrama Causal Microempresa, relación costos - materia prima.

El diagrama causal que se evidencia en la figura 5 hace referencia al análisis del costo del pedido sobre cada ítem de materia prima, su cantidad y el costo total de materia prima.

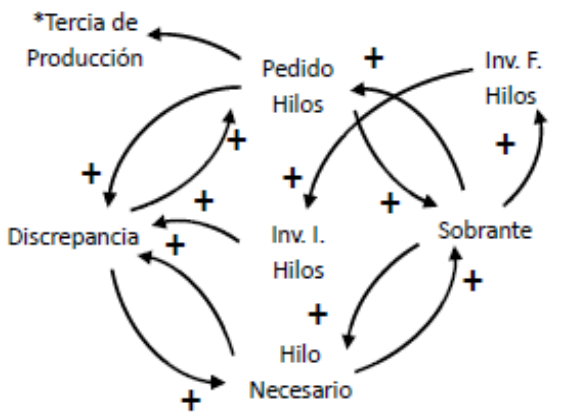

Figura 6. Diagrama Causal Microempresa, relación materias primas tercia de producción.

La cantidad de inventario que se tenga por cada tipo de materia prima deberá ser contabilizado para el planteamiento del modelo, información que deberá incluirse en el análisis de la tercia de producción. La figura 6 ilustra un claro ejemplo para la cantidad de hilos que se debe considerar para cada periodo de producción. Cabe mencionar que este último sistema o conjunto de variables se repite 16 veces más, acorde a las materias primas necesarias para la elaboración del producto. 


\section{b. Diagramas Forrester}

Después de haber elaborado el diagrama causal, se procedió a la construcción del diagrama Forrester ${ }^{2}$ (figura 7), que de igual forma que el causal se presenta por partes, para mayor entendimiento, y está unido por medio de variables fantasma, aquellas que están en líneas punteadas, para facilitar la visualización y lectura del diagrama.

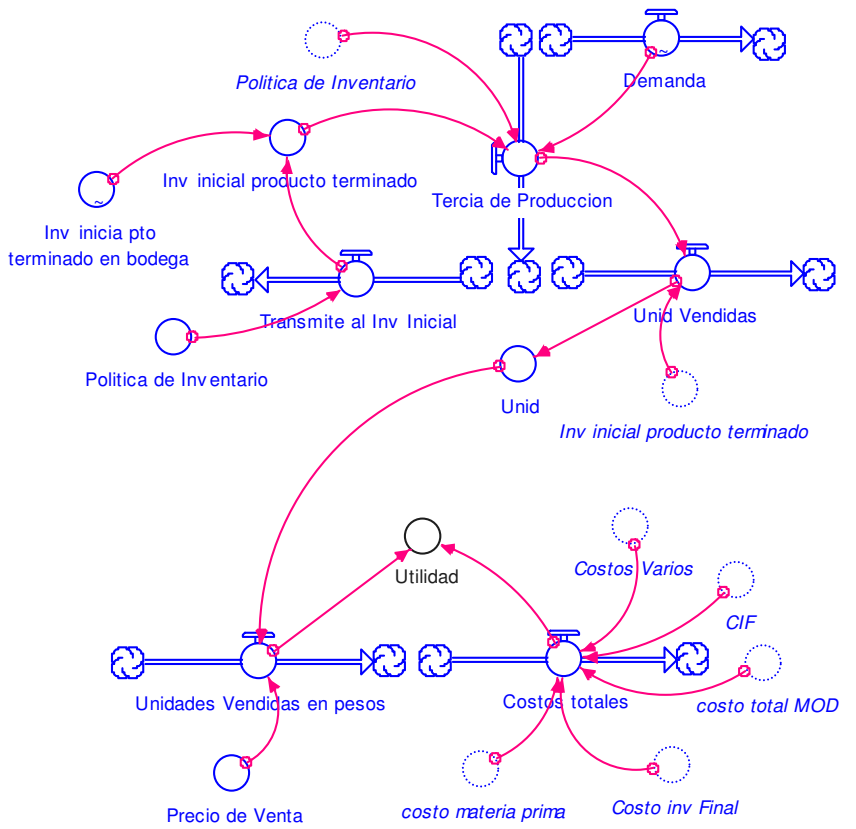

Figura 7. Diagrama Forrester Microempresa, relación tercia de producción - costos de producción

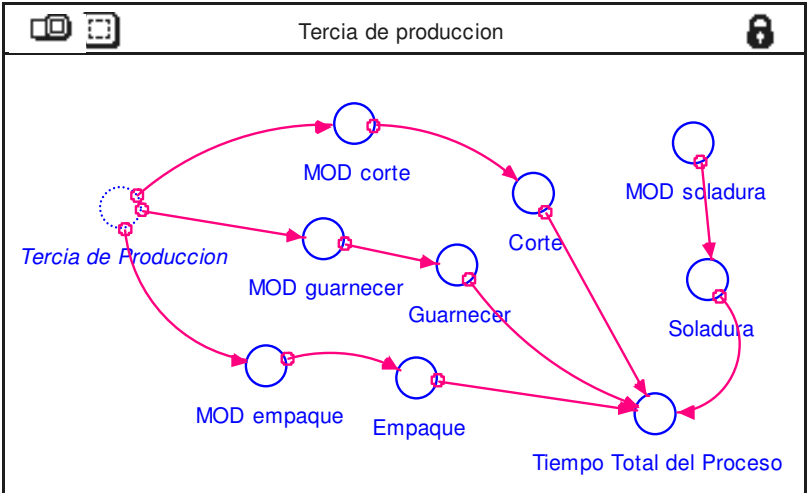

Figura 8. Diagrama Forrester Microempresa Calzado Wacky 2 parte (comportamiento de la mano de obra, según la tercia de producción).

\footnotetext{
${ }^{2}$ Diagrama de flujos y niveles. Los cuadros corresponden a niveles en donde se incorpora el estudio de variables que pueden cuantificar su aumento o disminución en un periodo de tiempo, dadas unas entradas o salidas del mismo (flujos). También contiene variables auxiliares, representadas por los círculos externos a los flujos, que contienen información detallada de cada variable que afecta de manera directa el comportamiento del sistema.
}

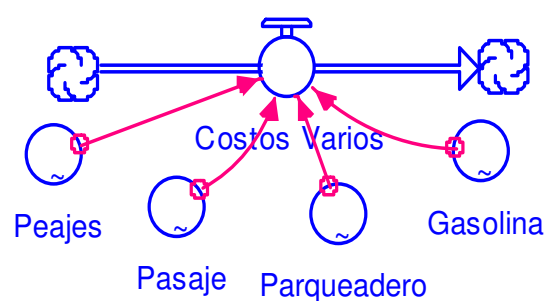

Figura 9. Diagrama Forrester Microempresa, costos varios.

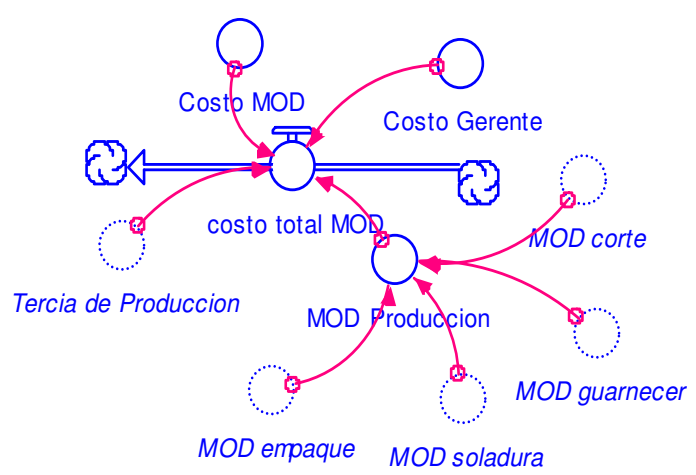

Figura 10. Diagrama Forrester Microempresa, relación costos directos de fabricación - tercia de producción

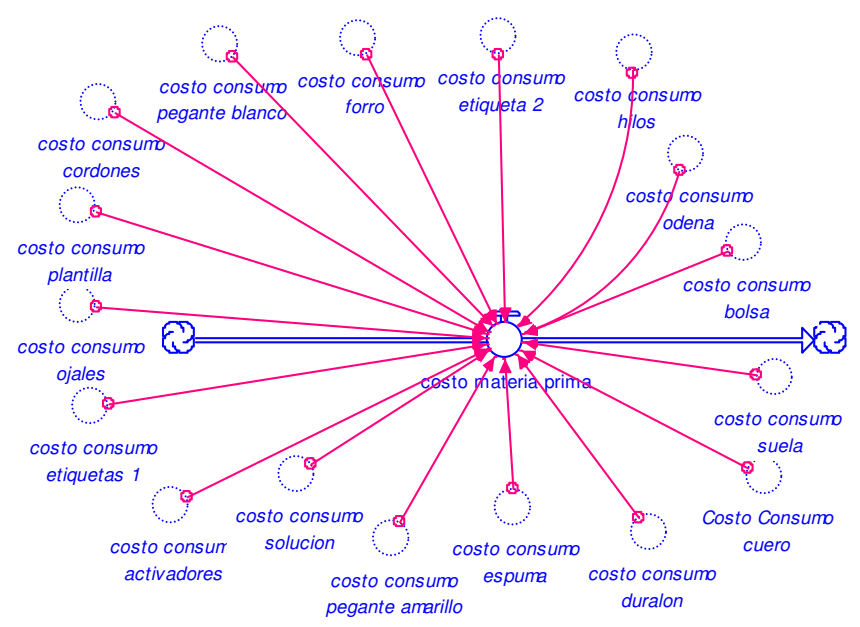

Figura 11. Diagrama Forrester Microempresa, costos de materias primas.
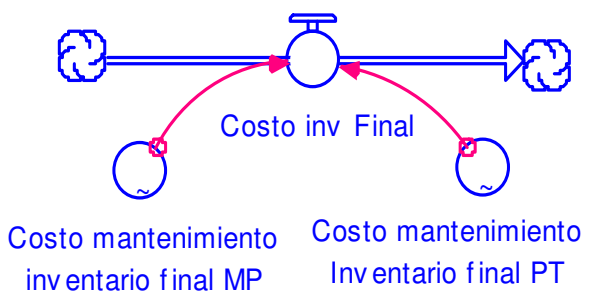

Figura 12. Diagrama Forrester Microempresa, comportamiento costo inventario final. 


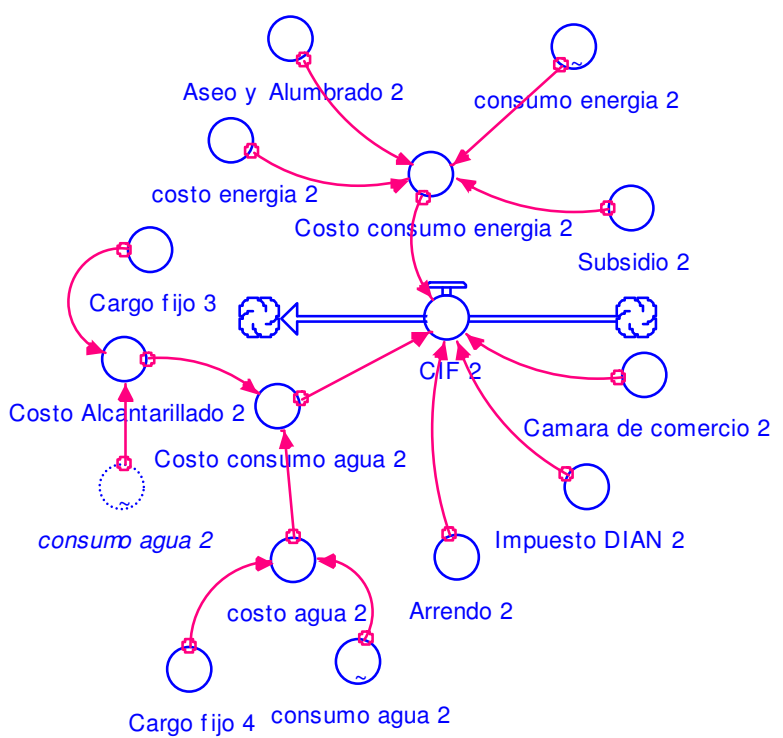

Figura 13. Diagrama Forrester Microempresa Calzado Wacky 3 parte (comportamiento costos $\mathrm{CIF}$ ).

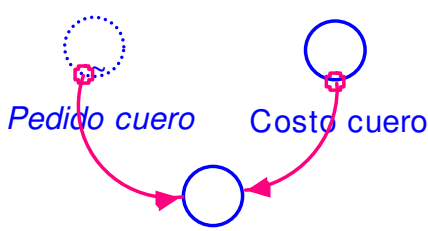

Costo Consumo cuero

Figura 14. Diagrama Forrester Microempresa Calzado Wacky 4 parte (el comportamiento de los costos de consumo de materia prima).

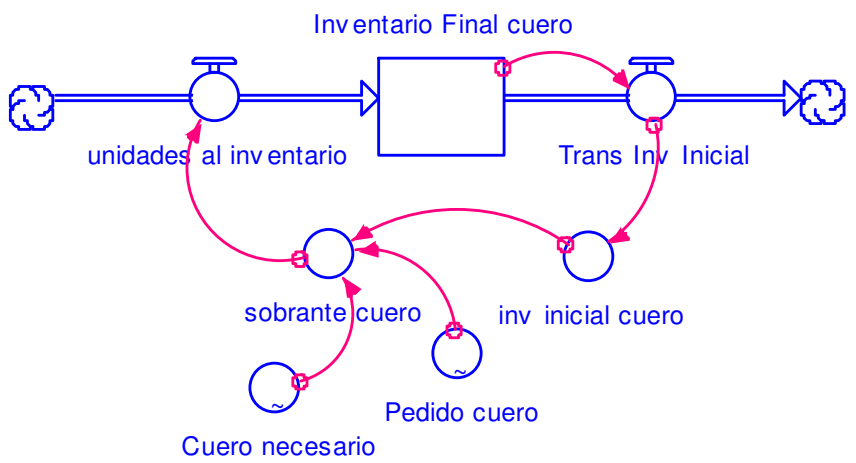

Figura 15. Sistema utilizado en la 5 parte del diagrama Forrester Microempresa Calzado Wacky.

Igualmente los dos últimos sistemas o conjunto de variables se repite 16 veces más, acorde a las materias primas necesarias para la elaboración del producto.

Una vez estudiado el comportamiento del sistema de producción de la microempresa, se procedió a identificar las variables críticas, sobre las que se pueden tomar acciones correctivas. Para ello se construyó el modelo de simulación para un periodo de 5 meses. La simulación se realizó en el software Stella ${ }^{\circledR} 9.0$, versión estudiantil.

\section{Validación del modelo}

La validez del modelo será realizada por 6 expertos, compuestos entre miembros del sistema productivo y personas con experiencia en este tipo de tema (Dinámica de sistemas) para determinar su validez, según los requerimientos del criterio de expertos propuesto por L. Campistrous y C. Rizo [4]. El análisis de este método arrojó como resultado un grado de calificación de "muy adecuado" frente al conocimiento de los expertos.

La técnica de Criterio de expertos propone las siguientes ventajas, según Mendoza [8]:

- En todo momento la información brindada por la persona más preparada en el tema, estará confrontada por los demás participantes.

- Se basa en la suposición de que varios expertos pueden llegar a un mejor pronóstico que una sola persona.

- No existe secreto y se fomenta la comunicación porque a veces los pronósticos y validaciones tienen influencia de factores sociales y pueden no reflejar un consenso.

- Como pronóstico visionario es una profecía que usa ideas y juicios personales, vinculados entre sí.

Los criterios de validación para el modelo planteado son:

1. Claridad Descriptiva: El modelo es comprensible a partir de las variables que lo integran y de las relaciones que se establecen entre ellas.

2. Integridad: El modelo se relaciona con las variables que permiten medir y tener información real $\mathrm{y}$ verídica de la capacidad de la microempresa Calzado Wacky.

3. Pertinencia: El modelo da respuesta a las necesidades que tiene la microempresa Calzado Wacky, para la medición de su capacidad.

4. Viabilidad: La aplicación del modelo es posible en las condiciones actuales del sector industrial.

5. Coherencia: Considera que el modelo constituye una herramienta válida para ayudar a potenciar la dimensión de capacidad de la microempresa Calzado Wacky.

De acuerdo con el procedimiento de la técnica, los resultados se exponen en la tabla 1, en donde se compara cada criterio, con un indicador base, (punto de corte). Cada uno de los criterios calificados por el grupo de expertos genera un resultado de "muy adecuado", lo que permite concluir que el modelo expuesto se ajusta al comportamiento del sistema productivo en la realidad en cuanto a la integridad, claridad descriptiva, pertinencia, viabilidad y coherencia. 
\begin{tabular}{|l|l|l|l|l|} 
Indicador & N-Promedio & Menor & Punto de corte MA & RESULTADO \\
\hline
\end{tabular}

\begin{tabular}{|c|c|c|c|c|}
\hline Claridad Descriptiva & $-0,2327$ & $<$ & 2,7920 & CUMPLE \\
\hline Integridad & $-0,2327$ & $<$ & 2,7920 & CUMPLE \\
\hline Pertinencia & $-0,2327$ & $<$ & 2,7920 & CUMPLE \\
\hline Viabilidad & 0,9307 & $<$ & 2,7920 & CUMPLE \\
\hline Coherencia & $-0,2327$ & $<$ & 2,7920 & CUMPLE \\
\hline
\end{tabular}

Tabla 1. Resultados validación por el Criterio de Expertos.

\section{RESULTADOS}

El planteamiento y desarrollo del modelo utilizó un alcance, vinculando variables como demanda y análisis de utilidad. Se consideraron medidas de desempeño como costos totales, capacidad de producción y de inventario.

\begin{tabular}{|l|l|}
\hline \multicolumn{2}{|c|}{ Descripción del Modelo } \\
\hline Nombre del sistema & $\begin{array}{l}\text { Sistema productivo Microempresa Calzado } \\
\text { Wacky }\end{array}$ \\
\hline $\begin{array}{l}\text { Objetivo del modelo del } \\
\text { sistema }\end{array}$ & $\begin{array}{l}\text { Estudiar el comportamiento de la } \\
\text { microempresa con base en su capacidad. }\end{array}$ \\
\hline Alcance Modelo & $\begin{array}{l}\text { Desde que se genera la demanda, hasta que } \\
\text { se obtiene la utilidad. }\end{array}$ \\
\hline $\begin{array}{l}\text { Medidas de desempeño del } \\
\text { modelo del sistema }\end{array}$ & $\begin{array}{l}\text { Utilidad del periodo } \\
\text { Costos Totales } \\
\text { Capacidad de Producción } \\
\text { Capacidad de inventario }\end{array}$ \\
\hline
\end{tabular}

Tabla 2. Descripción general del diseño del modelo de simulación Dinámica de Sistemas para la microempresa en estudio

El comportamiento de las medidas de desempeño general indican una clara diferencia entre los costos de producción totales, frente a las ventas. Ver gráfico 2.

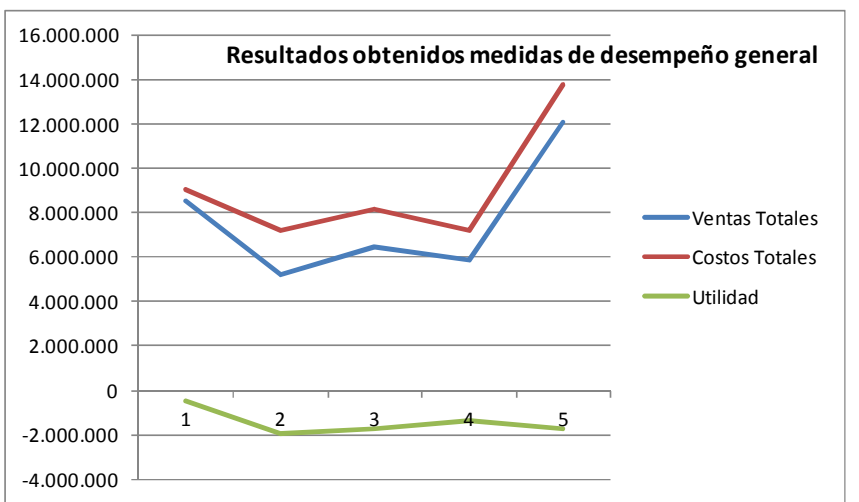

Gráfico 2. Resultados obtenidos del modelo de simulación. Medidas de desempeño general.

De acuerdo al análisis de los costos totales, se detalló en el diagrama causal cada uno de los ítems correspondientes a esta variable, por lo tanto se ilustra en el gráfico 3 el comportamiento, durante los 5 meses de simulación, de los costos de materias primas, de inventario final, indirectos de fabricación y costos varios. Se puede evidenciar una clara diferencia sobre los costos de materias primas, con un valor muy pronunciado en el quinto mes, y una tendencia creciente de los costos de inventario final.

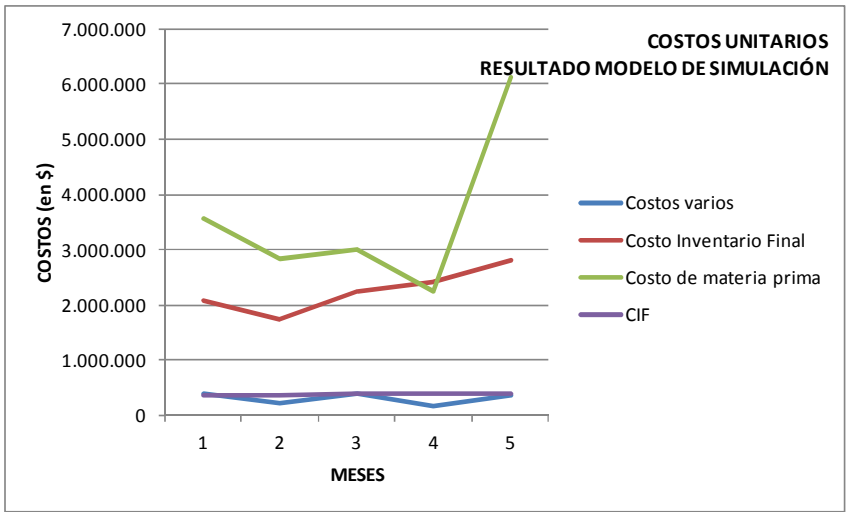

Gráfico 3. Resultados obtenidos del modelo de simulación. Medidas de desempeño de los costos relacionados.

Se debe recordar que al tener productos en inventario, estos se ven perjudicados por el riesgo de obsolescencia y deterioro, además de que el capital se ve reducido al tener poca circulación del producto.

\section{A. Escenarios de mejora.}

El estudio presentó dos escenarios de mejora para proponer un cambio que buscaba una disminución en los costos de materia prima, dado el análisis anteriormente expuesto.

\section{Reducción del almacenamiento de materias primas y del inventario de producto final}

Este escenario propone estudiar el comportamiento del sistema productivo con sus variables principales, teniendo en cuenta una reducción del $50 \%$ del almacenamiento en materias primas y en inventario final. Se puede contemplar un cambio en el valor de las utilidades para todos los meses de estudio, con un promedio de $\$ 1.444 .938$ por periodo. Este incremento se considera significativo, dadas las condiciones básicas en el cambio del modelo.

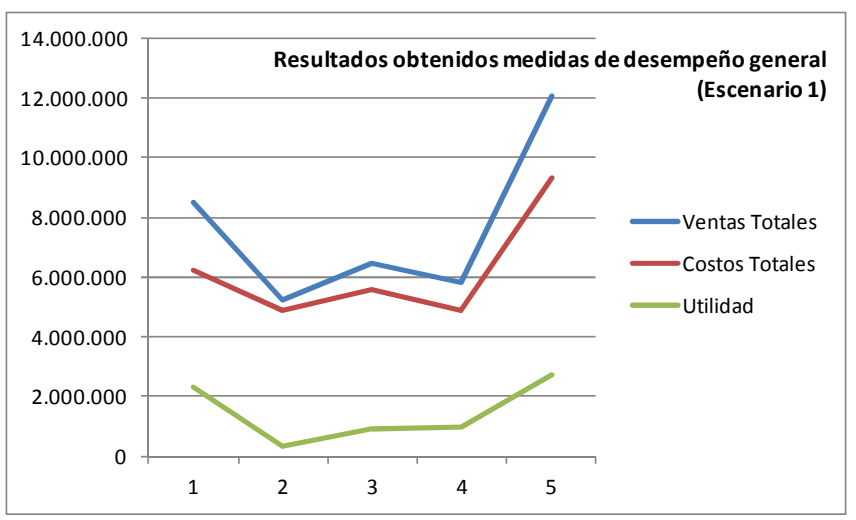

Gráfico 4. Resultados obtenidos. Desempeño general. Escenario 1. 
Los resultados de la gráfica 5 ilustran un cambio considerable en las ventas totales. El comportamiento de los costos discriminados se ilustra a continuación:

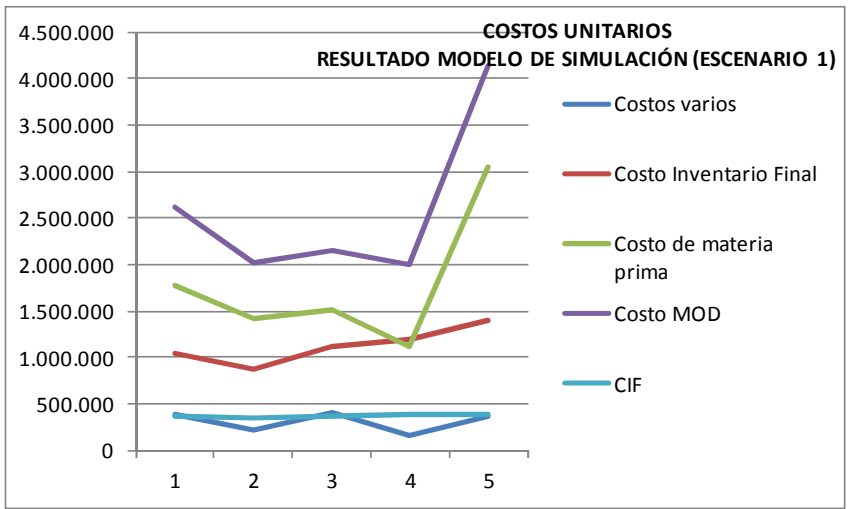

Gráfico 5. Resultados obtenidos. Desempeño por ítem de costos. Escenario 1.

El resultado de la reducción de los costos de materia prima se hace notorio, en cuanto se tome la decisión de controlar el almacenamiento de la materia prima. Aquí se considera importante sugerir a los administradores del proceso productivo la implementación de herramientas como el MRP (Material Requeriment Planning), que permite considerar la cantidad exacta de materias primas e insumos de acuerdo a un plan maestro de producción. También se sugiere contemplar la implementación de herramientas del Justo a Tiempo, en donde se pueda incluir la calidad y el tiempo de entrega de las materias primas para tener un desempeño integral.

\section{a. Adicionar un incremento en el precio de venta del $15 \%$}

Se decide estudiar el comportamiento del sistema productivo incrementando el $15 \%$ en el valor del precio de venta. Los resultados se ilustran en los gráficos 6 y 7 :

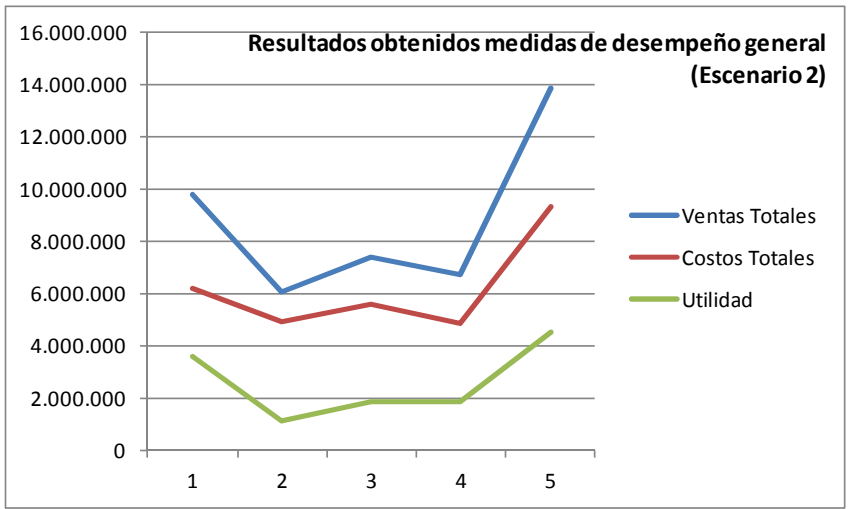

Gráfico 6. Resultados obtenidos. Desempeño general. Escenario 2.

Dado que los costos no incrementan significativamente por un cambio en el precio de venta, se debe aclarar que la cantidad a producir estimada para los 5 meses de estudio no varía, por lo que los costos unitarios corresponderían al mismo comportamiento del gráfico 5.

\section{CONCLUSIONES}

- La Dinámica de Sistema presenta una alternativa de simulación para sistemas complejos. El sistema productivo en estudio presenta una serie de desventajas en sus resultados de productividad, asociados a costos de producción. Surgió una propuesta de simulación del sistema productivo por medio de la herramienta, encontrando diferentes alternativas de mejoramiento en términos de la administración de materias primas y

- Dentro del modelamiento del sistema productivo de la microempresa de calzado, se pudo alcanzar el objetivo sobre la medición de su comportamiento en relación con su capacidad, logrando que el modelo se comporte se ajuste a la realidad, con el fin de proponer escenario de mejora para el beneficio de la microempresa.

- Con la ayuda de simulación mediante el Diagrama Forrester se pudo analizar de forma precisa y profunda, el sistema actual de la microempresa de calzado, obteniendo resultados sobre las fallas y aspectos positivos con respecto al comportamiento actual, resultados que sirven para realizar acciones de mejora, con el fin de contribuir a un mejoramiento en los niveles de productividad de la misma.

- $\quad$ Se diseñó y validó un modelo de simulación desde la metodología de Dinámica de Sistemas sobre el comportamiento de variables relacionadas con costos, cantidad y tiempos de producción. En este modelo se propone un esquema particular de análisis, teniendo en cuenta el comportamiento de las mismas en el sistema productivo de estudio.

- $\quad$ Según los resultados obtenidos, se consideró que la capacidad de inventario, tanto de materias primas como de producto terminado, como variable crítica es notable, ya que juega un papel crucial en la utilidad de la microempresa, teniendo en cuenta que en la simulación generada por Stella el alto índice de inventario está afectando el desempeño de la misma.

- Según los escenarios planteados, se percibe que al reducir las capacidades de inventario en términos monetarios en un $50 \%$, los costos se ven notablemente reducidos. Por lo que se puede concluir que este es uno de los factores que se debe mejorar tanto en condiciones de registro de inventario como en planeación de la producción.

\section{RECOMENDACIONES}

Queda pendiente para un futuro cercano, un trabajo de investigación profunda, para la elaboración del análisis costobeneficio, que en ultimas es lo que le interesa al gerente de 
toda empresa, observar cual sería la ganancia o los pros de realizar los cambios necesarios.

Lograr generalizar el modelo de simulación para estudiar el comportamiento de costos, tiempos y cantidades de producción de las microempresas de un sector específico en la ciudad de Tuluá, corresponde a un reto que en el mediano plazo se pretende abordar desde proyectos de investigación en la UCEVA.

\section{REFERENCIAS}

[1] AMAT DE SWERT, José María. (2009.). Modelo De Dinámica De Sistemas Para Implantación De Tecnologías De La Información En La Gestión Estratégica Universitaria. Universidad Politécnica de Catalunya. Cataluña. España.

[2] ARACIL, Javier. (2007). Dinámica de Sistemas, citado por Programa Ingeniería en Informática, Universidad Centroccidental Lisandro Alvarado. Octubre.

[3] BALLESTEROS RIVEROS, Diana Paola y BALLESTEROS SILVA, Pedro Pablo. Análisis de la productividad en el sector de las confecciones en Risaralda. En: Redalyc, Scientia Et Technica, diciembre, 2006. vol. XII, núm. 32.

[4] CAMPISTROUS P., L. y RIZO C., C. (2006).El criterio de expertos como método en la investigación educativa. Cuidad de la Habana.

[5] CHASE, Richard B, AQUILANO, Nicholas J, Administración de Operaciones: Producción y cadena de suministros, México 2009 pág. 555.

[6] CONGRESO LATINOAMERICANO Y ENCUENTRO COLOMBIANO DE DINÁMICA DE SISTEMAS. (2011). Medellín, Colombia. Editorial Universidad Pontificia Bolivariana.

[7] IÑAKI MORLÁN, Santa Catalina. (2010). Modelo De Dinámica De Sistemas Para Implantación De Tecnologías De La Información En La Gestión Estratégica Universitaria. Universidad del País Vasco. País Vasco. España.

[8] MENDOZA, Fernández Sandra Hurtado, criterio de expertos. su procesamiento a través del método Delphy, universitat de Barcelona, [en línea], Disponible en internet: http://www.ub.edu/histodidactica/index.php?option=com_cont ent\&view=article\&id=21:criterio-de-expertos-suprocesamiento-a-traves-del-metododelphy\&catid=11:metodologia-y-epistemologia\&Itemid=103 [9] PETER, Senge, et al. (2012). La Quinta Disciplina En La Práctica. Ediciones Granica S.A., Bueno Aires. 